\title{
La traducción literal del escritor Lu Xun (1881-1936) como impulso a la lengua vernácula (baihua) y a la nueva literatura en China
}

\author{
YU-FEN TAI 嗔繁势 \\ Tamkang University \\ yufen1116@hotmail.com
}

Recibido: 14 de octubre de 2011

Aceptado: 23 de febrero de 2012

\section{RESUMEN}

A partir de la mitad del siglo XIX, China empezó a enfrentarse a la hegemonía y la invasión de las potencias occidentales. Algunos intelectuales utilizaron la traducción como herramienta para introducir los estudios occidentales en China, sobre todo los de tecnología y filosofía, con el fin de favorecer la modernización de la sociedad. Otros intelectuales aprovecharon la traducción para estimular la evolución literaria y el paso hacia la lengua vernácula, el baihua. Entre ellos se encuentra el escritor Lu Xun (1881-1936), que defendia la traducción literal como motor para hacer evolucionar la lengua clásica hacia el baihua. Consideraba que mediante el baihua se podía lograr una literatura más cercana al pueblo y así educarlo. Al mismo tiempo, la traducción de Lu Xun conllevaba cierta manipulación ideológica del traductor. Las obras traducidas por él, en general, presentaban personajes sufridos y revelaban la injusticia social. Lu Xun quería que los chinos aprendieran de estas obras literarias. Por otro lado, desde una óptica contemporánea, la opinión de Lu Xun y su insistencia en adoptar una traducción literal encuentra un paralelismo en la extranjerización que defiende Lawrence Venuti a la hora de oponerse a una lectura "fluida”. Salvando las distancias, tanto Lu Xun como Venuti señalan que el exotismo puede acercar la cultura extranjera a la literatura meta y, en cierto sentido, evitar la hegemonía de cada época.

Palabras clave: Lu Xun, traducción literal, Venuti, extranjerización, historia de traducción en China.

Lu Xun's (1881-1936) conception of Literal Translation as impulse of vernacular Language (baihua) and new literature in China 


\begin{abstract}
From the mid-nineteenth century, China began to confront the hegemony and the invasion of Western powers. Some intellectuals in China used translation as a tool to introduce Western knowledge, especially studies of technology and philosophy, promoting the modernization of Chinese society. Other intellectuals took translation of foreign literatures as a literary evolution and attempted to enhance the importance of vernacular language, Baihua, in Chinese literature. Among the intellectuals, Lu Xun (1881-1936) believed that "literal translation" text was a method to promote the usage of vernacular language. By doing so, literature written in vernacular language instead of classical language could be more appreciated by the readership, and people could be further educated through reading these texts. At the same time, Lu Xun as a translator shows a certain degree of ideological manipulation. The selected works translated by Lu Xun generally focused on main characters who suffered social injustice. Through reading, Lu Xun wanted the Chinese readership to learn social issues from these literary works. In contemporary translation theory, the concept of "foreignization" raised by Lawrence Venuti also echoes "literal translation". Both Venuti and Lu Xun opposed the translation which follows the readership's reading habit in order to be more fluid and readable for them. The reason is that the translations with exotic flavor or unfamiliar words can create an impact and help the integration of foreign into local culture. Moreover, the "manipulation" of literal translation by Lu Xun is to promote the development of Chinese language and literature and to save the country as a mission. For Venuti, "foreignization" is a way to criticize the hegemony derived from English speaking culture and to be against the hegemonic domination.
\end{abstract}

Keywords: Lu Xun, literal translation, Venuti, foreignization, Chinese History of Translation.

Sumario: 1. Introducción: el trasfondo histórico y la traducción como herramienta ideológica para salvar al pueblo. 2. Lu Xun: la traducción como vehículo para transmitir conocimientos y educar al pueblo. 3. Lu Xun: la traducción literal como impulso a la lengua vernácula (baihua jerización (foreignization). 5. Referencias bibliográficas en español e inglés. Referencias bibliográficas en chino.

\title{
1. Introducción: el trasfondo histórico y la traducción como herramienta ideológica para salvar al pueblo
}

Desde la segunda mitad del siglo XIX, las potencias occidentales pretendieron "dividir" o "conquistar" China. La Guerra del Opio (1839-1842) entre China y Gran Bretaña imprimió una página negra en la historia de China. Después hubo nuevas guerras contra otros países extranjeros, entre ellos, Japón, Francia, Portugal, Rusia y Alemania, etc. La consecuencia de esas guerras no fue la victoria de China, sino su derrota, por lo que tuvo que ceder unos territorios y unos puertos marítimos a las potencias extranjeras, como es el caso de Hong Kong a Gran Bretaña y de Macao a Portugal. Al mismo tiempo, China se resignó a firmar tratados mediante los que indemnizaba a los países invasores. 
Bajo este trasfondo sociopolítico frustrante, los intelectuales chinos empezaron a reflexionar y plantearse cómo podrían salvar la nación. Surgieron algunas voces que clamaban por la reforma de la tradición y otras que coincidían en su mayor parte en un punto: el anhelo de modernización, encarnado en "lo extranjero". El deseo de occidentalización que albergaban los intelectuales otorgaba a la traducción un papel principal en la reconstrucción nacional. La traducción no tenía simplemente el valor que se le confería habitualmente:

[...] translation is not an isolated phenomenon. Rather, it is associated with certain major projects - nationalist, ideological and religious in nature [...] (Delisle y Woodsworth 1995: 25)

Aparte de transmitir una ideología sociopolítica, algunos escritores consideraban que mediante la traducción de literatura extranjera se podía impulsar la literatura y la lengua vernácula. De ahí que la traducción se convirtiera en el vehículo necesario para el fomento de la tradición literaria y para hacer de la lengua clásica o arcaica la fuente de nuevas transformaciones. A finales del siglo XIX y principios del XX en China, muchos intelectuales desempeñaban el doble papel de escritores y traductores en su orientación y en su labor creadora. Entre los escritores chinos, se encuentra el célebre Lu Xun. ${ }^{1}$ A continuación, vamos a tratar la figura de Lu Xun y su contribución a la literatura china moderna y a la lengua vernácula, el baihua, ${ }^{2}$ a través de su defensa de una traducción literal, que él mismo puso en práctica en su acto de traducir.

\section{Lu Xun: la traducción como vehículo para transmitir conocimientos y educar al pueblo}

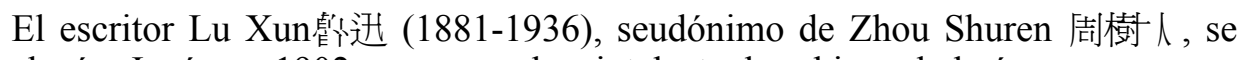
desplazó a Japón en 1902, como muchos intelectuales chinos de la época, para cursar estudios superiores de medicina. Después de las derrotas sufridas por China fren-

${ }^{1}$ Las obras literarias de Lu Xun que se han traducido al castellano son las siguientes:

Novelas escogidas. Trad. de Luis Enrique Délano. Pekín: Ediciones en Lenguas Extranjeras, 1972.

Grito de llamada. Trad. de Juan Ignacio Preciado y Miguel Shiao. Madrid: Alfaguara, 1978. 2009.

Contar nuevo de historias viejas. Trad. de Laureano Ramírez Bellerín. Madrid: Ediciones Hiperión,

Al catalán se han traducido las siguientes obras literarias:

Mala herba. Trad. de Marisa Presas y Séan Golden. Barcelona: Edicions 62, 1994.

Diari d'un boig $i$ altres relats. Trad. de Carles Prado. Barcelona: Edicions 62, 1984 y 2007.

En cuanto a sus obras sobre literatura y traducción, se han publicado los siguientes títulos en castellano:

Breve historia de la novela china. Trad. de Rosario Blanco Facal. Barcelona: Azul Editorial, 2002.

"Traducciones de traducciones". En: López García, D. (ed.). Teoría de la traducción: Antología de textos. Cuenca: Ediciones de la Universidad de Castilla-La Mancha, pp. 394-396.

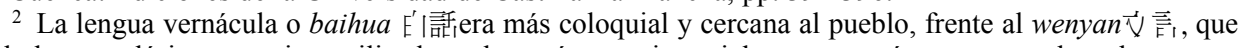
era la lengua clásica o arcaica, utilizada en los exámenes imperiales, que servían para acceder a los cargos oficiales. 
te a las potencias occidentales, llegó a la conclusión de que la literatura, sobre todo la extranjera, podía ser un instrumento para servir al país:

[...] la literatura puede estimular la personalidad y cambiar la sociedad. Por ello, es lógico que tenga la intención de introducir la literatura extranjera en China. ${ }^{3}$ (Xie y Cha 2004: 84)

Esta idea que tenía Lu Xun sobre la función de la literatura enlaza con las reflexiones de Even-Zohar, quien señala que "la literatura sirvió como factor omnipresente para la cohesión sociocultural" (1994: 360). La carrera literaria de Lu Xun empezaría con la traducción y giraría en torno a ella, aunque posteriormente sería recordado sobre todo como uno de los escritores más destacados de la literatura china del siglo XX. Para muchos críticos literarios, Lu Xun fue uno de los pioneros en la introducción de la literatura extranjera en China, una misión que llevó a cabo durante más de treinta años.

En este punto, debemos citar la influencia de otro intelectual, Liang Qichao 梁所帮 (1873-1929), que participó en la revolucionaria Reforma Wuxu4 龙成變洼 (1898), en el marco de una debilitada dinastía Qing. Lu Xun compartía con él la opinión de que la traducción podía permitir transformar la ideología del pueblo y, al mismo tiempo, educarlo. Basándose en esta idea, seleccionó algunas obras extranjeras en las que se describían los problemas e injusticias sociales y se reivindicaba la lucha por la patria para despertar el espíritu nacional (Zhang 2003: 4). A lo largo de su trayectoria como traductor, tradujo literatura japonesa, rusa, inglesa, rumana, checa, húngara, polaca, etc., hasta unas doscientas obras de escritores de unos veinte países (Xie y Cha 2004: 82).

Con el deseo de reformar una sociedad que consideraba atrasada abogó por una reforma social a través de sus traducciones y sus propios relatos, en los que aparecían personajes pertenecientes a las clases más desfavorecidas. Por medio de ellos, quiso transmitir su firme denuncia y a la vez difundir una "nueva literatura", más cercana al pueblo. Él, como la mayoría de traductores de finales del siglo XIX y principios del XX en China, aprovechó la traducción de la literatura extranjera para alcanzar el objetivo que se planteaba. Por ello, podemos decir que ese acto de traducir respondía a una cierta "manipulación", en el sentido que señala Theo Hermans al decir que "ninguna traducción es inocente": "From the point of view of the target literature, all translation implies a degree of manipulation of the source text for a certain purpose" (Hermans 1985: 11). La vocación de Lu Xun por la traducción literaria ha de entenderse bajo la perspectiva de transmitir unos conocimientos, pero también de canonizar la lengua vernácula. A continuación, hablaremos de la ideología de Lu Xun respecto a la traducción para impulsar el uso de la lengua vernácula y el inicio de la nueva literatura.

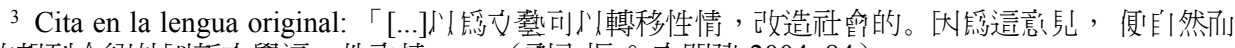

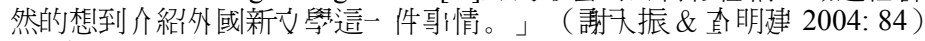

${ }^{4}$ La Reforma Wuxu duró poco más de cien días y fracasó. Con ella algunos intelectuales pretendían reformar los sistemas político, económico, educativo, militar, etc. para lograr que la nación fuese modernizada y pudiera enfrentarse a la amenaza de las potencias occidentales.
} 


\section{Lu Xun: la traducción literal como impulso a la lengua vernácula (baihua $\mathrm{F}^{\prime}$ 䎑) y la nueva literatura}

Según Lu Xun, mediante la traducción literaria se podía crear un nuevo modelo literario y también reestablecer la lengua vernácula, el baihua. En efecto, esta idea se convirtió en el lema de algunos escritores de la época, como Qu Qiubai 篦秋F́ (1899-1935), según el cual "la traducción puede servir para introducir las obras extranjeras a los lectores chinos, pero también tiene otra función, que es estimular la formación de una nueva lengua china moderna"5 (Qu en Liu 1995: 4). Partiendo de esta misma actitud, Lu Xun optó por una traducción literal para poner en práctica dicha teoría. En una carta de réplica al mencionado escritor, Lu Xun explicaba los motivos para adoptar una traducción literal. Ambos escritores compartían una misma afinidad por una traducción teñida del exotismo de la obra original:

Hasta hoy sigo siendo partidario de que una traducción debe ser fiel a la obra original aunque no resulte fluida en la lengua final. [...] Ante lo cual surge una pregunta: ¿por qué no optar por una traducción totalmente adaptada a la cultura china, que ahorre esfuerzos a los lectores? Mi respuesta es que eso también se puede considerar una traducción. Con esta traducción se pueden introducir nuevos contenidos y, al mismo tiempo, nuevas técnicas de expresión. ${ }^{6}$ (Lu Xun en Liu 1995: 13)

A partir de la idea de que la traducción debe ser fiel al original, Lu Xun enfatizaba la imitación del texto original como forma de introducir las estructuras gramaticales de la lengua extranjera para enriquecer la china. Su lema de que "una traducción debe ser fiel a la obra original aunque no resulte fluida en la lengua final" cobraría gran relevancia en la historia de la traducción en China.

Sin embargo, su insistencia en una traducción literal, a veces literal en exceso, podía dificultar la comprensión, debido a las ostensibles diferencias en la estructura gramatical entre las lenguas indoeuropeas y la china. Esta circunstancia despertó las críticas de algunos de sus contemporáneos, que definieron su estilo de traducción con términos como "traducción dura" y "traducción muerta". ${ }^{7}$ Algunos incluso llegarían a expresar abiertamente que sus traducciones eran difíciles de leer y de entender.

El propio Lu Xun reconoció que sus traducciones resultaban difíciles de leer, pero aun así pidió a sus lectores que siguieran leyendo, ignorando las frases "oscu-

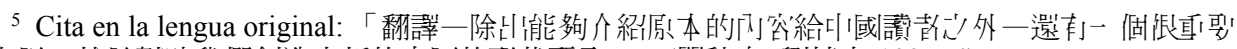

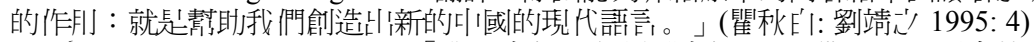

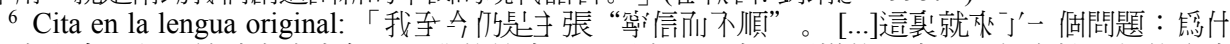

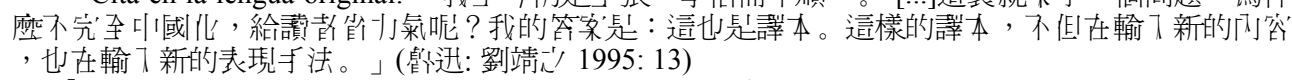

7 El término "traducción dura" en chino corresponde a 研臎, que también puede entenderse como "traducción forzada". Se trataba de una traducción marcada por las estructuras gramaticales de la lengua original, que evidentemente no resultaba fluida para los lectores del texto de destino. Por su parte, "traducción muerta" corresponde a 巽䛾 y se refiere a una traducción apagada, que despojaba de su vitalidad al texto original.
} 
ras" e incomprensibles (Liang en Lu Xun 1991: 76). Sin que las críticas parecieran importarle demasiado, Lu Xun afirmó que algunos nuevos usos lingüísticos empleados en su traducción literal (o dura) perdurarían en el tiempo y otros simplemente desaparecerían. En el fondo, todo su afán por defender una traducción literal se explica por el deseo de fortalecer la lengua vernácula para que sustituyera a la lengua clásica. Partiendo de algunos pasajes de sus traducciones, más adelante presentaremos algunos ejemplos que sirven para ilustrar hasta qué punto esta traducción dura crearía una cierta sensación de extranjerización en los lectores chinos. Pero antes vamos a citar a un intelectual de su tiempo, Liang Shiqiu (1903-1987), que escribió un artículo titulado "Ensayo sobre la traducción dura de Lu Xun"8 en 1929 para criticar con gran contundencia su estilo de traducción:

[...] ni siquiera se añade una palabra más, [el orden de las palabras es muy estricto], una no puede ir delante, la otra no puede ir detrás. A este modo [de escribir] se da el nombre de 'traducción"” [...]. ' (Liang en Lu Xun 1991: 74)

Para Lu Xun, si la lengua vernácula llegaba a sustituir a la lengua clásica, el pueblo podría acceder a los libros y con ello la educación llegaría a las masas, tras siglos fuera de su alcance. Esta ideología de una literatura del pueblo, de una literatura que perteneciera a todas las clases, no solamente a las más privilegiadas, se relacionaba también con la voluntad de modernizar la nación. Lu Xun pensaba que la lengua china debía adaptar estructuras gramaticales y elementos culturales procedentes de las lenguas extranjeras para estimular a la lengua china a convertirse en una lengua moderna. Con este ideario en mente, creó unas traducciones que ante todo se ajustaban a sus objetivos.

Así pues, la traducción literal de Lu Xun marcó una nueva página en la historia de la traducción en China, tanto para el ámbito lingüístico como el literario. Si bien es cierto que varios siglos antes ya se había aplicado un estilo de traducción literal para la adaptación de los textos budistas al chino, la contribución de Lu Xun se cifra en un orden muy distinto. La traducción literal defendida por Lu Xun posee una dimensión marcadamente política, con una clara voluntad de "manipulación" por parte del traductor que no poseían los textos budistas.

A continuación, vamos a citar varios ejemplos de las obras traducidas por $\mathrm{Lu}$ Xun para mostrar con mayor precisión en qué consistía su "traducción dura". Los pasajes en chino van acompañados por una traducción literal al español, para reflejar justamente hasta qué grado las frases en chino podían resultar poco comprensibles y un tanto enigmáticas para sus lectores.

Ejemplo 1:

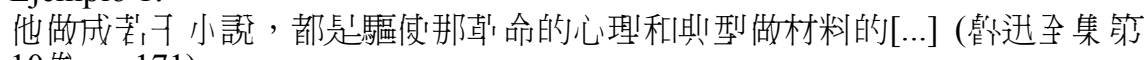
10巻: p. 171)

\footnotetext{
${ }^{8}$ En su título original 《諭管个迅先生的硬䛨》

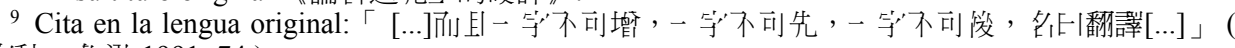
梁䈍偢：管㳡 1991: 74 ).
} 
Traducción "literal” al español: Él completa varias novelas, todas son para empujar a aquel espíritu y modelo revolucionario a crear un material [...] (Obras completas de Lu Xun, vol. 10: p. 171)

Ejemplo 2:

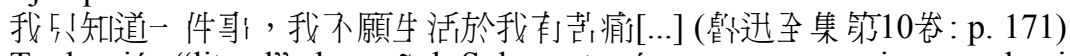

Traducción "literal" al español: Solamente sé una cosa: no quiero que la vida en mí sufra el dolor [...]. (Obras completas de Lu Xun, vol. 10: p.171)

Ejemplo 3:

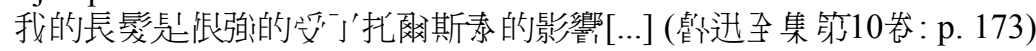

Traducción "literal” al español: Mi pelo largo ha recibido una fuerte influencia de Tolstoi [...]. (Obras completas de Lu Xun, vol. 10: p.173)

Ejemplo 4:

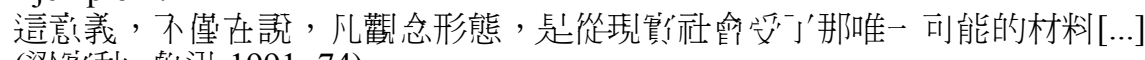

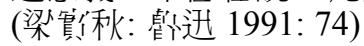

Traducción "literal" al español: Este sentido no sólo expresa cualquier ideología, desde la sociedad real se ha visto influido por un único material posible [...] (Liang en Lu Xun 1991: 74)

Ejemplo 5:

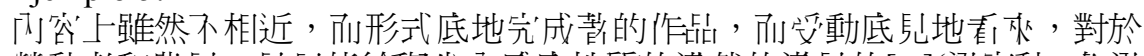

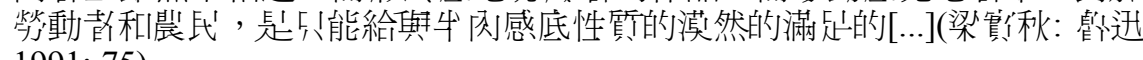
1991: 75)

Traducción "literal" al español: A pesar de que el contenido no es parecido, la forma de acabar la obra, en cuanto a su poder para conmover, sólo puede ofrecer a los trabajadores y los campesinos la mitad de sensualidad y una fría satisfacción [...]. (Liang en Lu Xun 1991: 75)

La traducción literal de Lu Xun aportaba toques claramente exóticos y extranjerizantes, que causaban incomprensión y extrañeza. Sin embargo, su insistencia en defender esta traducción literal en las circunstancias históricas que hemos descrito estaba dotada de un profundo significado. Los elementos lingüísticos extranjeros debían enriquecer la lengua autóctona y, paralelamente, se pretendía importar elementos de la literatura extranjera para insuflar una energía renovada a la literatura china. Este afán constante también le permitiría sacar partido de su labor como traductor en sus propias obras originales. Así, su primera novela, Diario de un loco

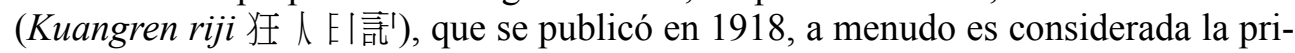
mera novela moderna china escrita en lengua vernácula, que marcaría el inicio de una nueva literatura china. Según algunos críticos literarios -aunque otros no opinan lo mismo-, en esta novela se aprecia la influencia de la obra de Gogol, que Lu Xun tradujo con interés. 


\section{Conclusiones. La preferencia por la extranjerización (foreignization)}

En primer lugar, resulta interesante hacer un análisis de las opiniones de Lu Xun y Lawrence Venuti respecto a la preferencia de ambos por la extranjerización. El concepto de traducción literal de Lu Xun parece aproximarse mucho al de extranjerización de Venuti. En un artículo de 1935, Lu Xun hablaba de su experiencia al traducir la obra rusa Almas muertas, de Gogol. Allí utilizaba conceptos similares a los que Venuti menciona sesenta años después en su libro The Translator's Invisibility: A History of Translation. En ese artículo, Lu Xun decía: "Antes de empezar a traducir, hay que enfrentarse a una pregunta: ¿esforzarse al máximo por conseguir una traducción domesticada o mantener el tinte exótico de la obra original?"10 (Lu Xun en Liu 1995).

Para Lu Xun, mantener el tinte exótico es un modo de acercarse a la obra original. ${ }^{11}$ Constatamos así que los conceptos de Lu Xun ante la traducción coinciden con los términos de domesticación y extranjerización de Venuti. Cabe recordar, como se sabe, que Venuti se vio influido por Schleiermacher, cuyo discurso en 1813 "Sobre los diferentes métodos de traducir" (Über die verschiedenen Methoden des Übersetzens) revelaba que una traducción puede seguir dos métodos: intentar un acercamiento "hacia el lector" o bien "hacia el autor". En realidad, a lo largo de la historia de la traducción, tanto en Occidente como en China, la cuestión de la traducción literal frente a la traducción libre ha representado una constante oposición binaria, aunque según las necesidades propias de cada época se ha optado por una u otra.

Volviendo a las figuras de $\mathrm{Lu}$ Xun y Venuti, a pesar de que los dos son partidarios de la extranjerización, cabe precisar que la intención de cada uno es distinta. Para $\mathrm{Lu}$ Xun, a través de la traducción extranjerizante se puede enriquecer y reformar la literatura y la lengua nacionales, como hemos mencionado anteriormente. En cambio, la intención de Venuti es criticar la hegemonía del inglés como lengua de llegada, de modo que "la traducción extranjerizante al inglés supone una resistencia contra el etnocentrismo, el racismo, el narcisismo cultural y el imperialismo" (Hurtado 2001: 618). Como contrapunto, podemos apuntar que Hatim y Mason no comparten esta concepción general de Venuti. Para ellos, la adopción de un método de traducción u otro depende de una situación dada, por lo que el modo de actuar del traductor se entiende como una manifestación de su propia ideología en un contexto determinado.

Thus, it is not domestication or foreignization as such which is 'culturally imperialistic' or otherwise ideologically slanted; rather, it is the effect of a particular strategy employed in a particular socio-cultural situation which is likely to have ideological implications. The translator acts in a social context and is part of that context. It is in this sense that translating is, in itself, an ideological activity. (Hatim y Mason 1997: 146)

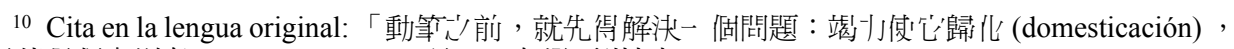

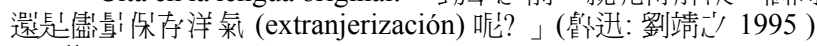

${ }^{11}$ La postura de Lu Xun ante la traducción literal se identifica con la tendencia apuntada por Munday: “'literal' now means 'sticking very closely to the original”". (2001: 33).
} 
La opinión de Hatim y Mason nos permite afirmar que la extranjerización de $\mathrm{Lu}$ Xun responde a una ideología concreta en el contexto sociopolítico de principios del siglo XX en China: salvar la nación e introducir elementos lingüísticos foráneos para impulsar el empleo del baihua.

Por otro lado, Venuti defiende que la figura del traductor ha de ser "visible", debido a la falta de reconocimiento de su papel a lo largo de la historia. Coincidimos con Venuti en la necesidad de que la función del traductor sea cada vez más "visible", puesto que la traducción siempre ha quedado relegada a la autoridad de la obra original. No es nuestro objetivo exaltar el valor de la traducción, pero no podemos negar que la visibilidad del traductor representa una forma de valorar su tarea. Tampoco podemos negar que todas las grandes obras de la literatura universal se han difundido a través de sus traducciones y que éstas han impulsado el desarrollo de las literaturas nacionales, como así lo evidencia la labor de Lu Xun.

Lu Xun no mostró una inclinación semejante a la de Venuti por destacar la función del traductor, pero habló de varios tipos de lectores, lo que representaba un concepto muy avanzado en su época. Para él, la traducción debía adaptarse a un tipo u otro de lectores. ${ }^{12}$ En este sentido, la traducción literal iba destinaba a unos lectores que habrían recibido una buena educación, es decir, a un grupo selecto. Con ello, podemos ver que Lu Xun era muy consciente de cómo llevar a cabo su tarea de traductor para introducir unos conocimientos entre un determinado público. La idea de que diferentes lectores requerían distintos tipos de traducción y que ésta obedecía a un objetivo es lo que la Escuela Funcionalista alemana definiría con la idea de escopo, la finalidad de la traducción:

No existe $\boldsymbol{l a}$ (única forma de realizar una) traducción de un texto; los textos meta varían dependiendo del escopo que se pretende alcanzar. (Reiss y Vermeer 1996:84)

Los traductores desempeñan un papel fundamental para la formación de las literaturas y las lenguas nacionales, como hemos intentado ilustrar en este breve análisis de la figura de Lu Xun como traductor. Con el desarrollo de los estudios de la traductología, la contribución de los traductores y las traducciones gradualmente ha sido más valorada.

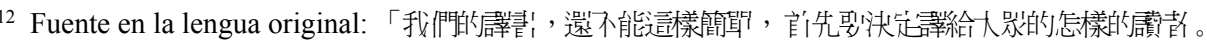

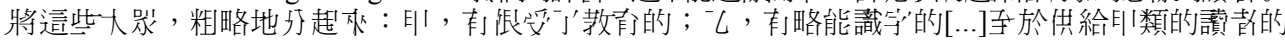

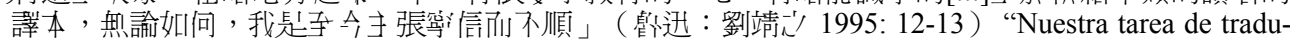
cir obras no resulta sencilla. En primer lugar, hay que decidir para qué público de lectores traducir. En general, éste se divide en: $a$ ) los lectores que han recibido una buena educación, $b$ ) los que saben leer [...] Respecto a las traducciones destinadas al primer tipo de lectores, hasta hoy sigo siendo partidario de que la traducción debe ser fiel a la obra original aunque no resulte fluida en la lengua final." (Lu Xun en Liu 1995: $12-13)$. 


\section{Referencias bibliográficas en español e inglés}

Carbonell i Cortés, O., Traducción y cultura: De la ideología al texto. Salamanca: Ediciones Colegio de España 1999.

Delisle, J. \& Woodsworth, J. (eds.), Translators through History. Amsterdam: John Benjamins 1995.

Even-Zohar, I., «La función de la literatura en la creación de las naciones de Europa», en: Villanueva, D. (ed.), Teoría de la literatura. Santiago de Compostela: Universidad de Santiago de Compostela 1994, 357-374.

Hatim, B. \& Mason, I., The translator as communicator. Londres: Routledge 1997.

Hurtado, A., Traducción y traductología: Introducción a la traductología. Madrid: Cátedra 2001.

Lefevere, A., Traducción, reescritura y la manipulación del canon literario. Salamanca: Ediciones Colegio de España 1997.

Munday, J., Introducing Translation Studies: Theories and Applications. Londres: Routledge 2001 .

Reiss, K. \& Vermeer, H. J., Fundamentos para una teoría funcional de la traducción. trad. de S. García Reina y C. Martín de León, Madrid: Ediciones Akal 1996.

\section{Referencias bibliográficas en chino}

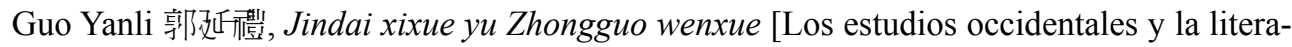
tura china en la era moderna]. Nanchang: Editorial Baihuazhou Wenyi 1999.

Liu Ching-Chi 製持-I/ (ed.), Fanyi lunji [Antología de ensayos en torno a la traducción]. Taipei: Bookman 1995.

Liang Shiqiu 梁旗偢, «Ensayo sobre la traducción dura de Lu Xun», en: Lu Xun, Lu Xun luzhan wenxuan [Antología de ensayos de Lu Xun]. Taipei: Editorial Xuexin 1991.

Lu Xun 髹迅, Lu Xun quanji [Las obras completas de Lu Xun]. vol. 10, Taipei: Editorial Gufeng 1989.

Lu Xun 礕迅, Lu Xun luzhan wenxuan [Antología de ensayos de Lu Xun]. Taipei: Editorial Xuexin 1991.

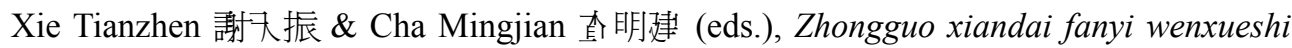
1898-1949 [Historia de la traducción literaria moderna en China 1898-1949]. Shanghai: Shanghai Foreign Language Education Press 2004.

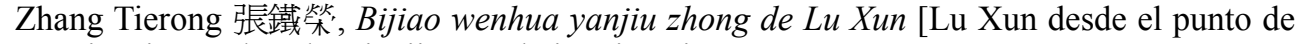
vista intercultural]. Tianjin: Nankai University Press 2003.

Zhang Zhongliang 掁战, Wusi shiqide fanyi wenxue [La traducción literaria en torno al Movimiento de 4 de Mayo de 1919]. Taipei: Editorial Xiuwei 2006. 